\title{
Superparamagnetic behaviour of metallic Co nanoparticles according to variable temperature magnetic resonance
}

Received 00th January 20xx, Accepted 00th January 20xx

Ilya V. Yakovlev, ${ }^{a, b, c}$ Stanislav S. Yakushkin, ${ }^{a}$ Mariya A. Kazakova, ${ }^{a}$ Sergey N. Trukhan, ${ }^{a}$ Zoya N. Volkova, ${ }^{d}$ Alexander P. Gerashchenko, ${ }^{d}$ Andrey S. Andreev, ${ }^{e}$ Arcady V. Ishchenko, ${ }^{a}$ Oleg N. Martyanov, ${ }^{a}$ Olga B. Lapina ${ }^{a, b}$ and Jean-Baptiste d'Espinose de Lacaillerie ${ }^{{ }^{*} \mathrm{c}}$

Investigating the size distributions of Co nanoparticle ensembles is an important problem, which has no straightforward solution. In this work, we use the combination of ${ }^{59} \mathrm{Co}$ internal field nuclear magnetic resonance ( ${ }^{59} \mathrm{Co}$ IF NMR) and ferromagnetic resonance (FMR) spectroscopies on a metallic Co nanoparticle sample with a narrow Co nanoparticle size distribution due to encapsulation within the inner channels of carbon nanotubes. High-resolution transmission electron microscopy (TEM) images showed that the nanoparticles can be represented as prolate spheroids, with the majority of particles having an aspect ratio between 1 and 2 . This observation has increased the accuracy of superparamagnetic blocking size calculations from Néel relaxation model by introducing the actual volume of the ellipsoids taken from the image processing. ${ }^{59} \mathrm{Co}$ IF NMR and FMR experiments conducted under different temperatures allowed us to observe the thermal blocking of superparamagnetic particles in full accordance with the TEM particle volume distribution. This proved that these magnetic resonance techniques can be used jointly for characterization of Co nanoparticles in the bulk of the sample.

\section{Introduction}

Cobalt nanoparticles and composite materials based on them are extensively studied and have already found applications in various scientific and industrial fields including catalysis $^{1-5}$, electromagnetic absorptive materials ${ }^{6,7}$, energy storage materials $^{8-10}$, and medicine ${ }^{11,12}$. The usage properties of an ensemble of Co nanoparticles depend on their size, morphology, crystalline and magnetic structures, properties that may actually all be connected to each other. They also depend on the particle size distribution of the ensemble and on interparticle interactions ${ }^{13-15}$. Thus, predicting the particles' morphology and crystalline structure in specific synthesis conditions is of crucial importance for their successful application.

The main characterization techniques that can be used for studying Co nanoparticles include X-Ray diffraction (XRD), transmission electron microscopy (TEM), scanning probe microscopies, magnetic resonance techniques (ferromagnetic resonance $(F M R)^{16,17}$ and ${ }^{59} \mathrm{Co}$ internal field nuclear magnetic resonance $\left({ }^{59} \mathrm{Co}\right.$ IF NMR) $\left.{ }^{18-22}\right)$. All these techniques have their advantages and disadvantages.

${ }^{a .}$ Boreskov Institute of Catalysis SB RAS, 5 Pr. Lavrentieva, 630090, Novosibirsk, Russia.

${ }^{b .}$ Novosibirsk State University, 1 Pirogova, 630090, Novosibirsk, Russia

c. SIMM, ESPCI Paris, Université PSL, CNRS UMR 7615, 10 Rue Vauquelin, 75005, Paris, France.

${ }^{d .}$ Mikheev Institute of Metal Physics UB RAS, 18 S. Kovalevskoi, 620990,

Ekaterinburg, Russia

e. Total Research and Technology Feluy (TRTF), Zone Industrielle C, 7181 Feluy, Belgium f. + Electronic Supplementary Information (ESI) available. See DOI: $10.1039 / x 0 x x 00000 x$

The application of XRD is limited for very small or defective nanoparticles due to diffraction pattern broadening; local microscopic techniques characterize a very small fraction of the sample, which is not always representative of its entirety. FMR and ${ }^{59}$ Co IF NMR techniques are particularly dependent on the particle size distributions. Small superparamagnetic particles give rise to a very sharp signal in FMR, sometimes making large ferromagnetic particles indistinguishable. On the contrary, ${ }^{59}$ Co IF NMR provides information only about particles large enough to be ferromagnetic and mostly about the biggest ones due to the volumetric origin of the signal and higher enhancement factor.

Recently, Liu et al. $^{22}$ have proposed a technique of temperature differential nuclear ferromagnetic resonance (TDFNR) that allows to "cut out" a narrow part of the distribution and study it separately by subtracting ${ }^{59}$ Co IF NMR spectra recorded at different temperatures. This technique is based on the thermally induced ferromagnetic to superparamagnetic transition. Above a given temperature, particles smaller than a certain critical size experience thermal "flips" of magnetization during the experimental time and become invisible by ${ }^{59} \mathrm{Co}$ IF NMR. However, to efficiently employ this technique for particles a few nanometers in size one needs to be able to achieve very low temperatures (liquid helium temperature), which may not be achievable with every experimental setup or too expensive due to the high price of liquid helium. Thus, we have turned our attention to another spectroscopic technique - ferromagnetic resonance (FMR). 

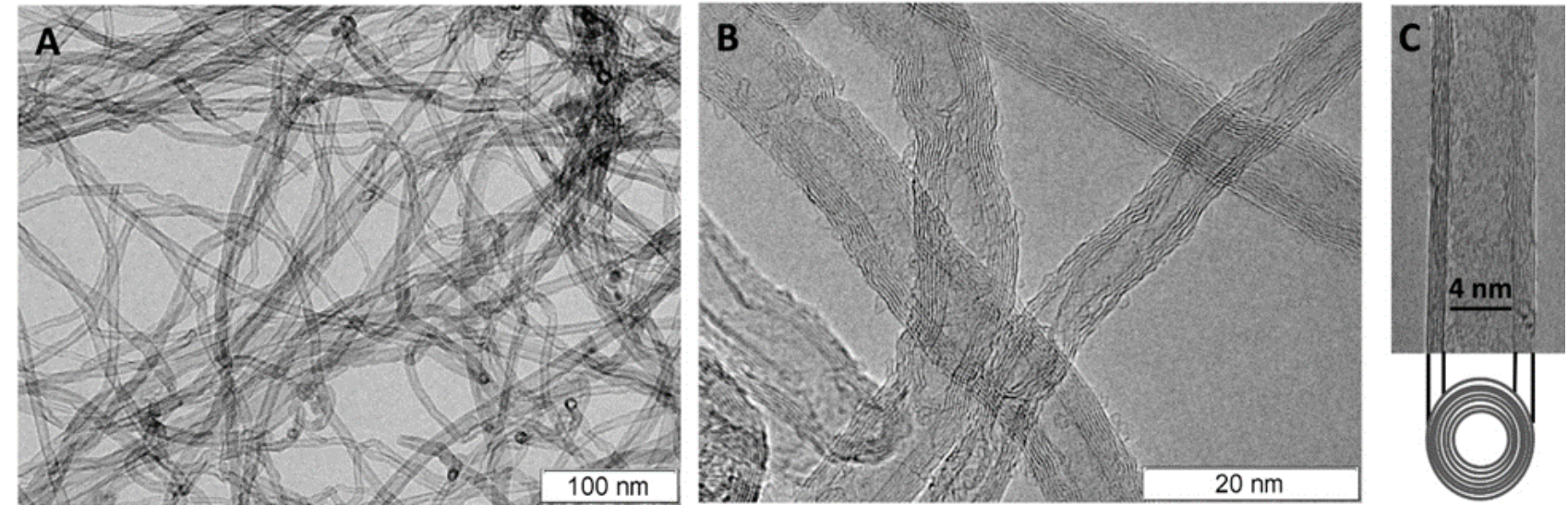

Figure 1. HRTEM micrographs of the oxidized carbon nanotubes (A-B) together with a schematic side view (C).

since in this case the electronic spin magnetization is probed directly.

In this work, we have attempted to simultaneously apply ${ }^{59}$ Co IF NMR and FMR to investigate Co nanoparticles contained inside multi-walled carbon nanotubes (MWCNTs) and establish the application limits of both methods. In our previous works ${ }^{23,24}$, factors which affect the formation of Co nanoparticles in the MWCNTs structure such as CNT dimensions, impregnation solution concentration and postsynthetic acidic treatment, were discussed in detail. Herein, we could thus concentrate on Co/MWCNT samples containing metallic Co nanoparticles localized inside the MWCNT channels. ${ }^{59}$ Co IF NMR experiments performed at room temperature and at $30 \mathrm{~K}$, and FMR experiments performed at elevated temperatures up to $600 \mathrm{~K}$, provided the data on the blocking temperatures from which the critical sizes for superparamagnetic behaviour of encapsulated particles could be estimated. These results were efficiently compared with the particle size distribution obtained using high-resolution TEM.

\section{Experimental}

\section{Co/MWCNT hybrid material synthesis}

Multi-walled carbon nanotubes with specific structural properties and morphology were synthesized using a wellestablished procedure already described in detail by some of us elsewhere ${ }^{25}$. In brief, the catalytic chemical vapor deposition technique was employed for the growth of nanotubes via pyrolysis of ethylene on the surface of Fe-Co catalyst at $680{ }^{\circ} \mathrm{C}$. The needed morphology of MWCNTs was obtained by using $\mathrm{Al}_{2} \mathrm{O}_{3}$-supported catalyst with $30 \mathrm{wt} . \%$ of the active component. The catalyst residues were removed by boiling the obtained MWCNTs in a solution of $15 \%$ hydrochloric acid for 4 hours with continuous stirring. After that, the MWCNT samples were washed with distilled water until the suspension reached a neutral $\mathrm{pH}$ value. The purity of MWCNTs obtained after the acid purification was $99.7 \%$ (as determined by X-ray fluorescence analysis). After that, the nanotubes were treated with concentrated nitric acid for 2 hours to create openings and defects on their surface in order to facilitate Co deposition.

According to high-resolution transmission electron microscopy (HRTEM) data (Fig. 1), the obtained nanotubes were built up of 5-7 carbon monolayers with the inner and outer diameters (ID and OD, respectively) of the tubes amounting to approximately $3.9 \mathrm{~nm}$ and $7.2 \mathrm{~nm}$ correspondingly. Using the method of acid-base titration described by Boehm ${ }^{26}$ the content of $\mathrm{COOH}$ groups in oxidized MWCNT was determined and measured to be 2.7 units per $\mathrm{nm}^{2}$. The specific surface area of oxidized MWCNTs was 360 $\mathrm{m}^{2} / \mathrm{g}$.

Co nanoparticles were deposited inside the MWCNTs by incipient wetness impregnation with an aqueous solution of $\mathrm{Co}\left(\mathrm{NO}_{3}\right)_{2} \cdot 6 \mathrm{H}_{2} \mathrm{O}(98 \%$, Sigma-Aldrich) and subsequent reduction under $\mathrm{H}_{2}$ flow. The procedure for preparing of $\mathrm{Co} / \mathrm{MWCNT}$ samples was described in detail earlier ${ }^{23,24}$. The resulting sample contained 4.2 wt.\% of Co (as determined by X-ray fluorescence analysis). For further studies by ${ }^{59} \mathrm{Co}$ IF NMR and FMR, the reduced samples were transferred to ampoules that were sealed without contact with air immediately after the reduction procedure.

\section{Characterization techniques}

BET surface area was measured by nitrogen adsorption at $77 \mathrm{~K}$ using an ASAP-2400 Micromeritics instrument.

$X$-ray fluorescent analysis was performed on an ARL Perform'X (Thermo Scientific) equipped with a rhodium anode. Elemental content was estimated using a standardless UniQuant procedure $^{27}$.

High-resolution transmission electron micrographs (HRTEM) for statistical analysis were recorded using JEM-2010 microscope (Jeol, Japan) with acceleration voltage of $200 \mathrm{kV}$ and spatial resolution of $0.194 \mathrm{~nm}$. HRTEM data was analyzed using FIJI software package. 


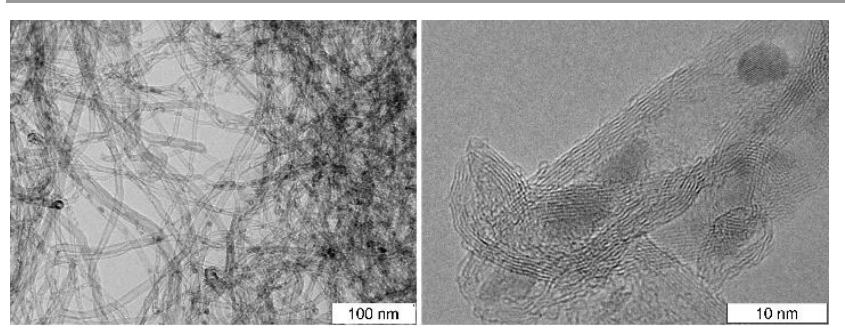

Figure 2. HRTEM micrographs of the Co/MWCNT hybrid material taken at different magnifications. Co nanoparticles are visible as dark grey areas.

${ }^{59}$ Co Internal Field Nuclear Magnetic Resonance ( ${ }^{59}$ Co IF NMR) experiments were performed using a Bruker Avance III spectrometer and a home-built probe in the absence of external magnetic field. The experiments were conducted at $30 \mathrm{~K}$ and $300 \mathrm{~K}$ to differentiate the signal of the particles according to their blocking temperature ${ }^{28}$. A custom-built auto-tuning device was used to avoid the time-consuming procedures of manual tuning and matching. A $\theta-\tau-\theta$ echo sequence was used for broadband excitation with a $\theta$ pulse length of $1 \mu \mathrm{s}$ and a $6 \mu$ s interpulse delay. The echo signals were recorded varying the carrier frequency stepwise each $500 \mathrm{kHz}$ to cover the entire spectral width. After performing Fourier transform, phase and baseline corrections, the real parts of obtained echo signals were integrated. The results of the integration were then assigned as the intensities of the signal at each frequency. The spectra were also recorded with several different power levels of RF irradiation according to the established procedure for obtaining quantitative ${ }^{59} \mathrm{Co}$ IF NMR spectra ${ }^{29}$. The variation of temperature did not cause any significant $Q$ factor change due to the fact that the FWHM of any individual Fourier-transformed echo remained constant between $30 \mathrm{~K}$ and $300 \mathrm{~K}$.

Ferromagnetic resonance (FMR) spectra were recorded using an X-band Bruker EleXsys 500 spectrometer equipped with ER $4114 \mathrm{HT}$ high-temperature cavity with digital temperature control unit BVT3000. Continuous-wave mode was employed with 4 Oe modulation at frequency of $100 \mathrm{kHz}$. For the FMR experiments, the sample was sealed in a quartz ampoule with
$2 \mathrm{~mm}$ inner diameter after the reduction. With this diameter, the $Q$ factor decrease due to conductivity of the sample remains negligible. Heating of the sample also led to a $Q$ factor decrease of less than $5 \%$. Considering this, the precision of quantitative measurements was satisfactory for all measurements.

\section{Results and Discussion}

\section{High-resolution transmission electron microscopy}

Figure 2 shows a HRTEM micrograph recorded for the Co/MWCNT hybrid material. Remarkably, all of the Co nanoparticles in the sample were found inside the carbon nanotubes. This means that the acidic treatment of the nanotubes creates enough inner channel openings for efficient Co ion penetration. The fact that the Co nanoparticles are located inside the inner channels implies the presence of strong capillary forces during Co impregnation. Since the particle shapes in the sample are not spherical, we have analysed the micrographs using a bivariate particle size distribution. The resulting distribution of particles over their long axis (C) and their short axis (A) as well as the distribution of their aspect ratios $(C / A)$ is given in Figure 3 . According to these data, the median aspect ratio of Co nanoparticles was equal to $\sim 1.6$. Thus, the particles in the sample were slightly elongated, confirming previous qualitative observations ${ }^{24}$.

HRTEM data proved that the Co particles were not spherical, thus we propose using a non-spherical model for the correct determination of particle volumes. Indeed, because of the axial symmetry imposed by the confinement within the carbon nanotubes, they can be represented as prolate spheroids. Taking this into account results in a realistic particle volume distribution (Fig. 4), which is the physically relevant property when considering effects related to magnetic ordering of the material.

\section{${ }^{59}$ Co IF NMR and FMR}

The ${ }^{59}$ Co IF NMR response of a ferromagnetic particle depends on its size among other factors. In large multi-domain particles,
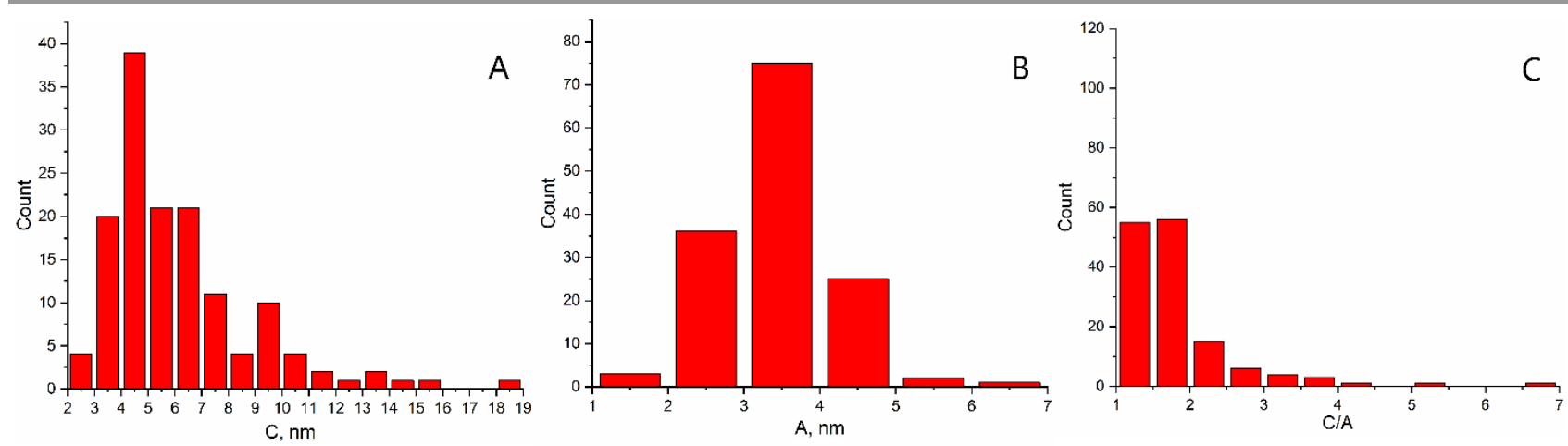

Figure 3. Left - distribution of particle long axes (C), middle - distribution of particle short axes (A), right - distribution of particle aspect ratios (C/A).

Co nuclei located inside the domains and domain walls experience two different mechanisms of RF field enhancement leading to a drastic increase of the signal originating from and near domain walls ${ }^{30,31}$. When a Co particle is small enough that the energy barrier of domain wall creation becomes too high, the conservation of single magnetic domain becomes more 
energetically favorable (a commonly reported value for the multi-/single-domain limit at RT is $70 \mathrm{~nm}$ for a spherical particle ${ }^{32}$ ).

The resonance frequency of Co inside a single domain particle increases with respect to the one of Co in a multidomain particle, due to the presence of uncompensated demagnetization field, which is aligned parallel to the hyperfine field ${ }^{33}$. Finally, when the size of the particle is small enough that its magnetic anisotropy energy becomes comparable to the thermal energy, the characteristic time of random "flips" of the magnetization that occur due to thermal fluctuations may become smaller than the characteristic time of the experiment (usually tens of microseconds in NMR). That leads to a complete loss of NMR signal, since the spin quantum states at the start of the experiment and at its end are different (thus averaging the magnetization to zero during the time of experiment).

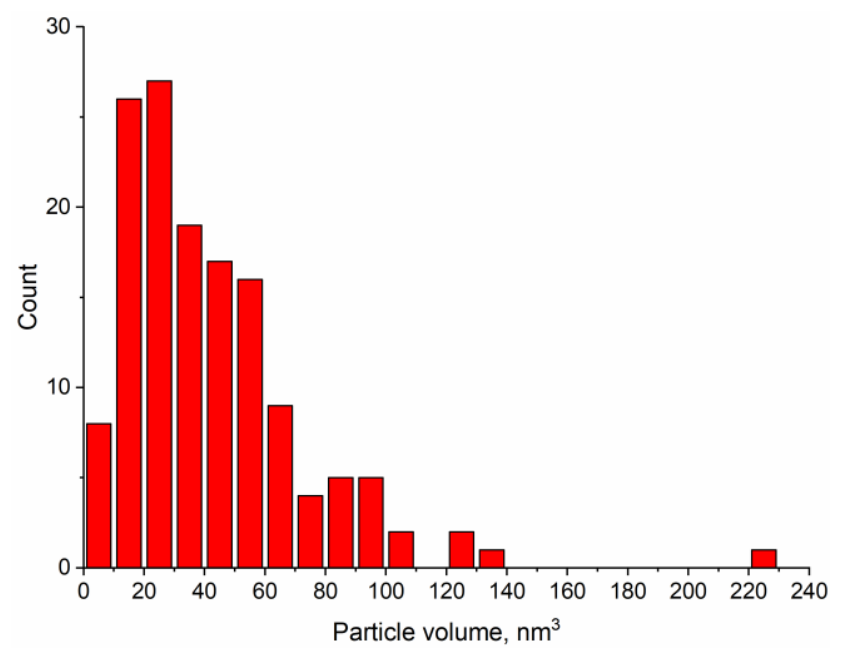

Figure 4. Co nanoparticle volume distribution obtained from the experimental $\mathrm{A}$ and $\mathrm{C}$ axes distributions provided by HRTEM.

Such state of particles is called superparamagnetic, and the critical volume $\mathrm{V}$ of the particle, above which its magnetization becomes "blocked" during the experimental observation time at a given temperature $T_{B}$ is described by the Arrhenius-type Equation (1) originally proposed by $\mathrm{Néel}^{34}$ :

$$
K V=k_{B} T_{B} \ln \left(\tau^{\tau_{m}} / \tau_{0}\right)
$$

where $k_{B}$ is the Boltzmann constant, $K$ is the anisotropy constant of the material $\left(5^{*} 10^{6} \mathrm{erg}^{*} \mathrm{~cm}^{-3}\right.$ for hcp Co), $\tau_{\mathrm{m}}$ is the characteristic measurement time, and $\tau_{0}$ is the characteristic "flipping attempt" time (usually considered to be between $10^{-9}$ and $\left.10^{-12} \mathrm{~s}\right)^{35}$. The volume dependence of the blocking temperature expressed in Eq (1) is the basic principle underlying the determination of particle size distribution by ${ }^{59}$ Co IF NMR. Any given temperature corresponds to a critical volume above which particles are ferromagnetic. ${ }^{59} \mathrm{Co}$ IF NMR signal intensity at a given temperature is thus proportional to the total volume of particles larger than the corresponding critical volume among other factors. Consequently, collecting the ${ }^{59}$ Co IF NMR spectra at different temperatures allows reconstructing in principle a volume size distribution. This procedure is based on the assumption that Equation (1) derived for spherical particles constitutes a valid approximation when the particles aspect ratio is not too high (as opposed for example to particles in $^{24}$, where their high aspect ratio provides for a considerable magnetic shape anisotropy).

The fact that ${ }^{59}$ Co IF NMR depends on particle sizes can be illustrated by comparing the room temperature ${ }^{59} \mathrm{Co}$ IF NMR spectra of three samples with similar Co content but different particle sizes (Fig. S2). In the sample of the present study (ID $\approx$ $4 \mathrm{~nm} \mathrm{~nm}, \mathrm{OD} \approx 7 \mathrm{~nm}$ ), the Co nanoparticles are located inside carbon nanotube channels and are thus limited in size. The nanotubes of higher OD ( 9 and $18 \mathrm{~nm}$ ) contained the bigger Co nanoparticles located inside the inner channels ( $I d \approx 4$ and 7 $\mathrm{nm}$, respectively) and on the outer surface. For the smallest $\mathrm{OD}=7 \mathrm{~nm}$, the majority of the Co particles were undetectable by ${ }^{59}$ Co IF NMR as their state is superparamagnetic at RT and at the NMR timescale. The NMR intensity was thus considerably smaller than for the ones in MWCNT of bigger OD. Indeed, in the approximation of spherical particles in the absence of substantial magnetic shape anisotropy using a characteristic time for ${ }^{59}$ Co IF NMR experiment of about $20 \mu \mathrm{s}$ and $\tau_{0}$ of $10^{-10} \mathrm{~s}$, we can estimate from Eq. 1 the room temperature superparamagnetic limit (critical diameter of a spherical particle corresponding to the blocking volume $\mathrm{V}$ ) to be $D_{\text {crit }}=5.8 \mathrm{~nm}$.

The ${ }^{59}$ Co IF NMR spectrum taken at $30 \mathrm{~K}$ displays a much higher intensity and was shifted toward higher frequencies compared to the room temperature spectrum (Fig. 5). Bloch's law for spontaneous magnetization explains such increase of resonance frequency, while the intensity increased according to Boltzmann distribution. However, after taking both of these factors together with the change of $T_{2}$ relaxation time (for more information on $\mathrm{T}_{2}$ measurement see Supplementary) into account for a quantitative comparison of signal intensities, the amplitude of the $30 \mathrm{~K}$ spectrum at $216.5 \mathrm{MHz}$ (resonant frequency of single-domain fcc Co particles) remained about 40 times higher (Fig. 5, red). This can be explained by the additional superparamagnetic Co particles that have undergone blocking into ferromagnetic state with the decrease in temperature. 


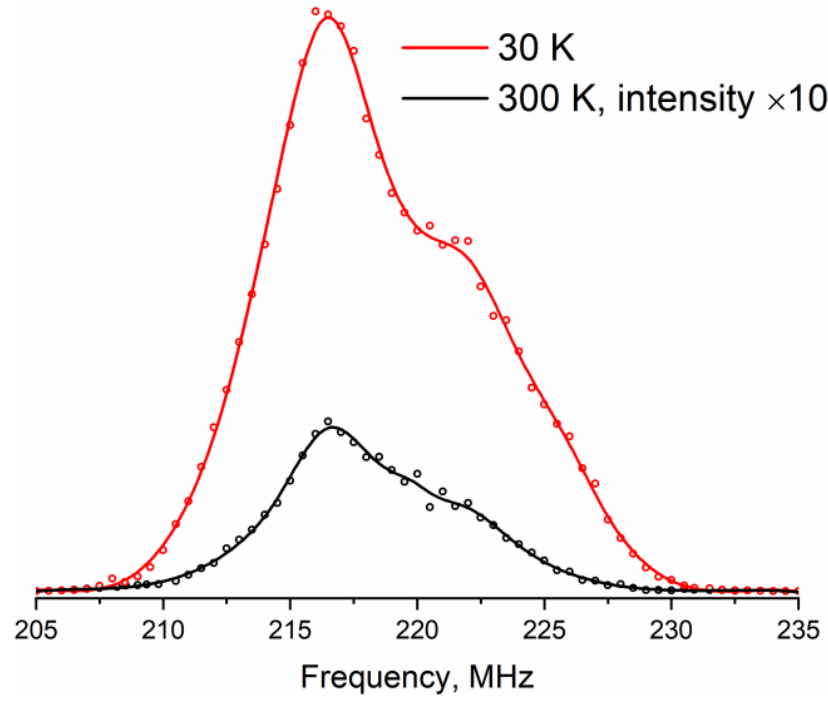

Figure 5. ${ }^{59} \mathrm{Co}$ IF NMR spectra of Co/MWCNT material recorded at room temperature (black, intensity multiplied by a factor of 10 for clarity) and $30 \mathrm{~K}$ (red) corrected for the temperature dependence of resonance frequency and amplitude. Experimental points are depicted with circles; interpolated lines are drawn for clarity. The $30 \mathrm{~K}$ spectrum is shifted into the lower frequency by about $5 \mathrm{MHz}$ to account for the resonance frequency shift caused by the increase of the spontaneous magnetization according to the Bloch's law. The spectral intensities were corrected to account for Curie's law and relaxation time difference.

At $30 \mathrm{~K}$, the estimate of the critical particle size given by Eq. 1 is $10 \mathrm{~nm}^{3}$. However, the actual critical volume may be somewhat bigger than estimated from Eq. (1) due to magnetic anisotropy associated with the slight non-sphericity of the particles and surface effects. To account for these morphological effects, Liu et al. ${ }^{22}$ have used an empirical 2.5 multiplier on the diameter for the critical blocking size predictions in order to match them to TEM observations.

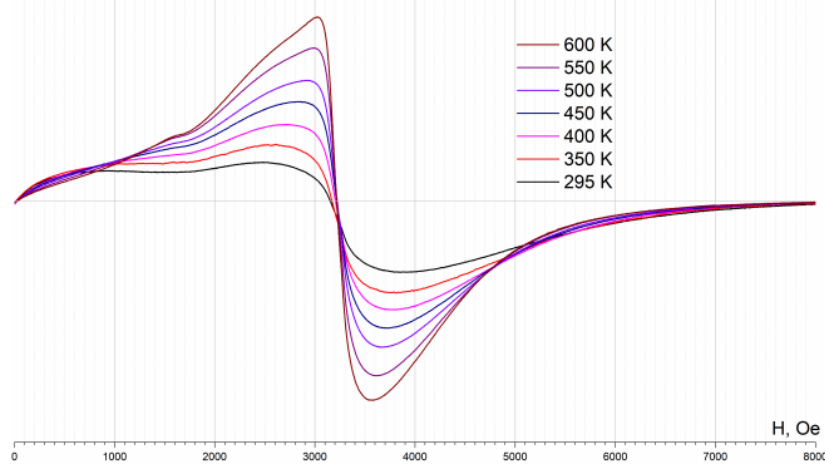

Figure 6. FMR spectra of $\mathrm{Co} / \mathrm{MWCNT}$ hybrid material taken at various temperatures. The intensity of the resonant components of the signal grows due to gradual unblocking of ferromagnetic particles and due to narrowing of the signal caused by temperature increase.

However, in our work, the critical size limits seemed to coincide well with the results for particle size distribution obtained by TEM, i.e. the particles with volumes between 10 and $100 \mathrm{~nm}^{3}$ (which should become "blocked" in ${ }^{59}$ Co IF NMR experiment with decrease in temperature from $300 \mathrm{~K}$ to $30 \mathrm{~K}$ ) correspond to the major part of cobalt volume determined from the HRTEM data. Therefore, we assumed that the increase of signal intensity did indeed arise from magnetic blocking of the majority of nanoparticles in the sample. Thus, for the considered aspect ratios (Fig. 3), the spherical assumption remained valid.

Apart from particle size considerations given by the analysis of spectra intensity, ${ }^{59}$ Co IF NMR also provides the information on the magnetic and crystalline structures of the particles. In line with previously published assignments ${ }^{21}$, the spectrum recorded at $30 \mathrm{~K}$ exhibited a typical line shape, consisting of a Pseudo-Voigt profile at $216.5 \mathrm{MHz}$ that corresponds to single-domain fcc-Co particles and a complex line shape corresponding to hcp-Co phase (219-225 MHz). The latter line could also correspond to stacking faults in fcc-Co packing since they are indistinguishable from hcp-Co. This together with the magnetocrystalline anisotropy makes the line corresponding to hcp-Co much wider compared to fcc$\mathrm{Co}^{36,37}$. No well-defined resonances at $213 \mathrm{MHz}$ corresponding to domain walls occurring in multi-domain Co particles were present in the spectrum, which is not surprising considering the particle size distribution. A decomposition of the spectrum into a Pseudo-Voigt line corresponding to fcc-Co and the remaining signal obtained by subtracting the fcc-Co line from the spectrum resulted in roughly $60 \%$ fcc-Co content. The spectrum recorded at RT displayed a much weaker intensity resulting in a less reliable decomposition (possible decompositions are shown in Fig. S3). Nonetheless, the fcc-Co intensity was visibly lower compared to the $30 \mathrm{~K}$ spectrum, which is consistent with the fact that hcp packing stability increases with the nanoparticle size increase ${ }^{38}$.

In order to further investigate superparamagnetic particles in the sample, a series of ferromagnetic resonance (FMR) spectra at room and elevated temperatures were recorded (Fig. 6). At room temperature, there was a broad asymmetric signal in FMR spectrum, with two notable features. The absorption in the high-field region (2000-5000 Oe) can be attributed to the single-domain ferromagnetic nanoparticles interacting with the microwave radiation. The absorption by ferromagnetic particles in the low-field region (0-1000 Oe) is often considered non-resonant. Thus, the origin of that part of the signal is rather complex and difficult to simulate.

With the temperature increase, both the low-field and high-field signals changed. A narrow intense line appeared in the high-field region at 3200 Oe starting from $400 \mathrm{~K}$. When the temperature rose, the high-field line narrowed, followed by a low-field signal intensity decrease. These effects resulted from the superparamagnetic behaviour of Co nanoparticles: with the temperature increase, more nanoparticles exhibited superparamagnetic behaviour resulting in the increase of the narrow component. Simultaneously, the signal in the low-field region decreased since temperature fluctuations unblocked the magnetic moments of nanoparticles.

To analyze the superparamagnetic transition of the Co nanoparticles with the temperature change, the high-field part of the signal was simulated (Fig. 7) as a superposition of two Lorentzian-shape absorption lines: a narrow one, corresponding to "unblocked" nanoparticles (the double 
integral is called $\mathrm{I}_{\text {unblocked}}$ ), and a broad one corresponding to "blocked" nanoparticles (the double integral is called I blocked $_{\text {) }}$. The double integral of the narrow component is growing together with the temperature increase, supporting the idea of superparamagnetic nature of the signal (Fig. S4).
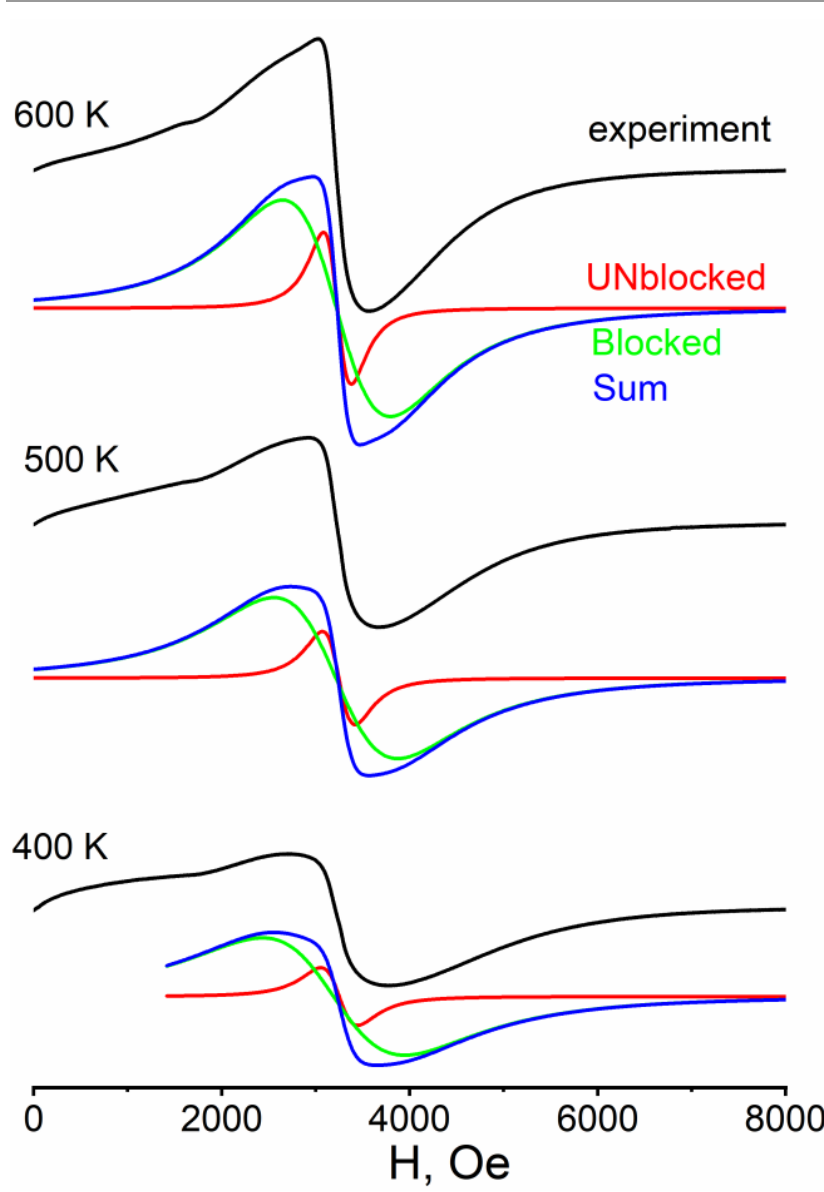

Figure 7. Experimental FMR spectra (black) at several chosen temperatures along with the simulated lines (shown in color). Red line represents the unblocked superparamagnetic nanoparticles; green line represents the blocked ferromagnetic particles; blue line is the sum of two simulated lines.

\section{Critical size calculations}

Based on the $I_{\text {unblocked }} / I_{\text {blocked }}$ ratio from the experimental FMR spectra, it is possible to choose the cut-off volume $(x)$ such that the ratio between the total volumes of particles with $V<x$ and of particles with $V>x$ is equal to $I_{\text {unblocked }} / I_{\text {blocked }}=$ 0.05 , the ratio observed in FMR at $600 \mathrm{~K}$. The obtained value of $x$ in this case is $17 \mathrm{~nm}^{3}$. Thus, the critical volume $V$ of a Co nanoparticle for FMR spectroscopy at $600 \mathrm{~K}$ is $17 \mathrm{~nm}^{3}$. Since the FMR experiments were performed using $\mathrm{X}$-band spectrometer, the characteristic measuring time $\tau_{m}$ can be estimated as $10^{-10} \mathrm{~s}$. These experimental values provided an estimate of the thermal relaxation time $\tau_{0}$ using the Neel equation (Eq. 1) as:

$$
\tau_{0}=\tau_{m}(F M R) * \exp \left(\frac{-K V}{k_{B} T_{B}}\right)=3.55 * 10^{-11} S
$$

which is in reasonable agreement with the commonly reported values of $10^{-9}$ to $10^{-12} \mathrm{~s}^{35}$.

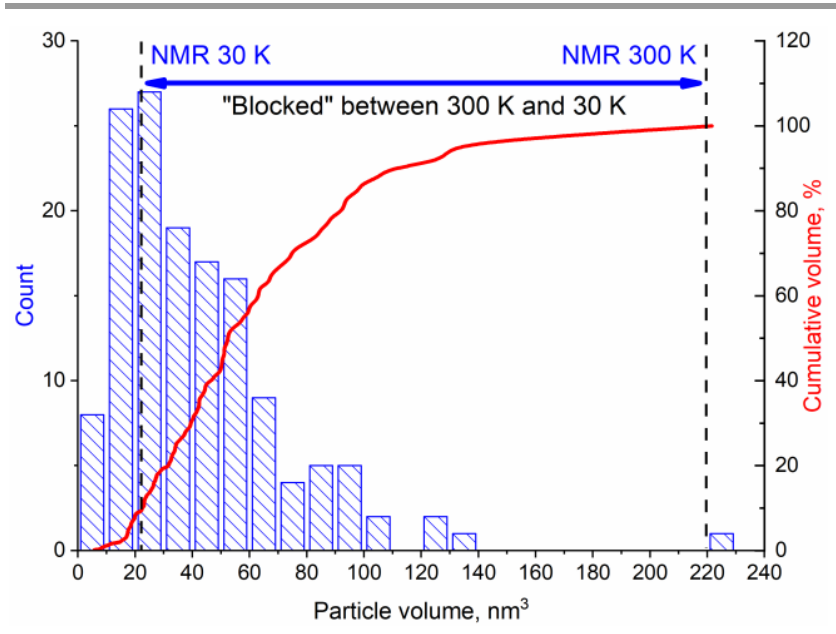

Figure 8. Depiction of particle blocking range for IF NMR between $300 \mathrm{~K}$ and $30 \mathrm{~K}$. Blue bars represent the HRTEM volume distribution. Solid red line is the cumulative volume.

Furthermore, this value of the critical volume of $17 \mathrm{~nm}^{3}$ observed at $600 \mathrm{~K}$ (FMR, 600K) allowed us to predict using Néel equation (1) the value of the critical volume at 300 $\mathrm{K}(\mathrm{FMR}, 300 \mathrm{~K})$ as:

$$
V(F M R, 300 K)=\mathrm{V}(F M R, 600 K) *\left(\frac{300 K}{600 K}\right)=9 \mathrm{~nm}^{3}
$$

Then, following the same logic using (Eq. 1) and using the thermal relaxation time $\tau_{0}$ obtained by $F M R$, we can estimate the critical volumes for ${ }^{59} \mathrm{Co}$ IF NMR spectroscopy at $300 \mathrm{~K}$ $V(\mathrm{NMR}, 300 \mathrm{~K})$ and $30 \mathrm{~K} V(\mathrm{NMR}, 30 \mathrm{~K})$ as follows:

$$
\begin{gathered}
V(N M R, 300 K)=\mathrm{V}(F M R, 300 \mathrm{~K})\left(\frac{\ln \left(\tau_{N M R} / \tau_{0}\right)}{\ln \left({ }^{\tau_{F M R} / \tau_{0}}\right)}\right)=220 \mathrm{~nm}^{3} \\
V(N M R, 30 \mathrm{~K})=V(N M R, 300 \mathrm{~K}) *\left(\frac{30 \mathrm{~K}}{300 \mathrm{~K}}\right)=22 \mathrm{~nm}^{3}
\end{gathered}
$$

The obtained values of $\mathrm{V}_{\text {crit }}$ for NMR spectroscopy differ from the predictions made earlier $\left(10 \mathrm{~nm}^{3}\right.$ at $30 \mathrm{~K}$ and $100 \mathrm{~nm}^{2}$ at $300 \mathrm{~K}$ ) by a factor of about 2 , but nonetheless, this range still covers the major part of the sample volume as determined from the cumulative volume graph (Fig. 8). According to these values, particles corresponding to $89 \%$ of total Co volume become thermally blocked in the $30 \mathrm{~K}-300 \mathrm{~K}$ temperature range, which is consistent with the significant intensity increase on the ${ }^{59}$ Co IF NMR spectra.

While for magnetic properties the physically relevant size is the particle volume, for most nanoparticle applications, for example supported heterogeneous catalysis, the size of interest is the linear size or the particle diameter. We thus also derived from the HRTEM data an effective spherical diameter distribution (Fig. 9) using:

$$
D_{E f f}=\sqrt[3]{A^{2} C}
$$

Obviously, the particle volume varies as the cube of the effective diameter so that the later parameter is much less sensitive and the discrepancy of a factor 2 in the volumes translates in a difference of only $25 \%$ in the effective 
diameters. This value was significantly lower than the $250 \%$ factor used by Liu et al. on Fischer-Tropsch catalysts ${ }^{22}$. In our case, the combination of FMR and ${ }^{59}$ Co IF NMR performed thus satisfactorily to characterize all Co particles from the linear size distribution given by HRTEM images analysis. This might be due to the better defined spheroidal geometry of the particles under consideration.

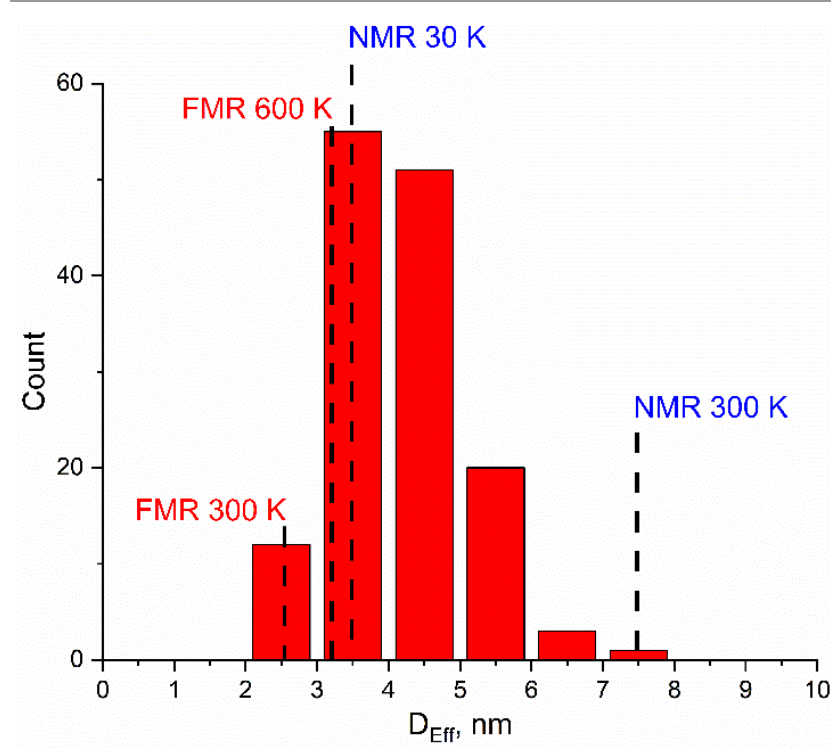

Figure 9. Recalculated spherical model Co nanoparticle $D_{\text {Eff }}$ size distribution obtained from the experimental $A$ and $C$ axes distributions provided by HRTEM. Dashed lines represent the critical particle sizes for FMR and ${ }^{59}$ Co IF NMR calculated from the obtained critical volumes.

At first glance, ${ }^{59}$ Co IF NMR and FMR provide very similar information on the Co nanoparticles in the sample. However, these techniques are very different by their nature. ${ }^{59} \mathrm{Co}$ IF NMR allows observation of particles solely in ferromagnetic state, and its operational range is limited to temperatures below room temperature due to inherently low sensitivity and long experimental time. This means that all spherical particles bigger than approximately 6-7 $\mathrm{nm}$ contribute to the ${ }^{59} \mathrm{Co}$ IF NMR spectrum taken at room temperature. The main advantage of this technique is its capacity not only to analyze particle sizes, but also to probe the crystalline and magnetic structure of the particles.

On the other hand, both ferromagnetic and superparamagnetic particles give rise to FMR signal making this technique less particle size selective. However, distinguishing the signals in FMR is not obvious. Moreover, an imprecision of thermal relaxation time $\tau_{0}$ determination at different temperatures may cause the discrepancy between the predicted and experimentally determined values of blocking sizes for ${ }^{59}$ Co IF NMR. Nevertheless, the sensitivity of FMR can be three orders higher compared to ${ }^{59} \mathrm{Co}$ IF NMR since it is the spin magnetization that is probed in this case. This facilitates doing FMR experiments at elevated temperatures, which extends the range of reachable critical blocking sizes to some extent. The probed blocking size range is indeed the main obstacle for these techniques due to a very weak dependency of the linear blocking size on the temperature (cubic root) and even weaker dependency on the measurement time (cubic root of logarithm).

\section{Conclusions}

Based on the image analysis by HRTEM, Co/MWCNT hybrid material possesses a relatively narrow size distribution of metallic Co nanoparticles with most of the particles being less than $6 \mathrm{~nm}$ in effective diameter. This was the result of particle confinement inside the channels of nanotubes that was observed on HRTEM micrographs. According to the difference between ${ }^{59} \mathrm{Co}$ IF NMR spectra recorded at $30 \mathrm{~K}$ and $300 \mathrm{~K}$, fcc crystal structure is stabilized in Co nanoparticles smaller than 6 $\mathrm{nm}$.

Using this sample, we have demonstrated that despite some experimental drawbacks, ${ }^{59}$ Co IF NMR and FMR are two complementary techniques that in combination provide a very powerful and, most importantly, bulk-averaged method for structural characterization of a large range of Co particle volume distributions based on HRTEM data analysis. Due to its high sensitivity, FMR can be used for particle size distribution investigation even at elevated temperatures, which increases the number of particles in superparamagnetic state. At the same time, ${ }^{59}$ Co IF NMR is a technique that provides unique information on the crystalline and magnetic structure of the particles. Moreover, the relatively long experimental time in ${ }^{59}$ Co IF NMR allows putting Co nanoparticles into superparamagnetic state in a much wider size range compared to FMR. However, these techniques possess drawbacks that limit the accuracy of critical blocking size determination, namely, very low signal-to-noise ratio in ${ }^{59}$ Co IF NMR and presence of non-resonant absorption signal in FMR spectra that leads to somewhat inaccurate decomposition of spectra. Furthermore, because the blocking temperature does not depend only on size but also on shape, caution must be used when discussing particle sizes using ${ }^{59} \mathrm{Co}$ IF NMR and FMR without using supplementary non-bulk techniques such as HRTEM.

\section{Conflicts of interest}

There are no conflicts to declare.

\section{Acknowledgements}

The authors are grateful for the support of RFBR grant № 2033-90184, and a budget project of Boreskov Institute of Catalysis. I.V.Y. acknowledged the French Embassy in Moscow for a PhD fellowship. Pascal Scholzen (ESPCl Paris) is thanked for rich discussions on ${ }^{59} \mathrm{Co}$ IF NMR.

\section{References}

1 M. O. Kazakov, M. A. Kazakova, Y. V. Vatutina, T. V. Larina, Y. A. Chesalov, E. Y. Gerasimov, I. P. Prosvirin, O. V. Klimov and A. S. Noskov, Catal. Today, 2020, 357, 221-230 
R. V. Jagadeesh, K. Murugesan, A. S. Alshammari, H. Neumann, M.-M. Pohl, J. Radnik and M. Beller, Science, 2017, 358, 326-332. V. Iablokov, S. A. Alekseev, S. Gryn, I. Bezverkhyy, V. Zaitsev, L. Kovarik, T. Visart de Bocarme and N. Kruse, J. Catal., 2020, 383, 297-303. F. Michalek, A. Lagunas, C. Jimeno and M. A. Pericàs, J. Mater. Chem., 2008, 18, 4692. X. Li, C. Zeng, J. Jiang and L. Ai, J. Mater. Chem. A, 2016, 4, 7476-7482.

M. A. Kazakova, N. V. Semikolenova, E. Y. Korovin, S. I. Moseenkov, A. S. Andreev, A. S. Kachalov, V. L. Kuznetsov, V. I. Suslyaev, M. A. Mats'ko and V. A. Zakharov, Russ. J. Appl. Chem., 2018, 91, 127-135.

D. Zhang, F. Xu, J. Lin, Z. Yang and M. Zhang, Carbon N. Y., 2014, 80, 103-111.

M. A. Kazakova, D. M. Morales, C. Andronescu, K. Elumeeva, A. G. Selyutin, A. V. Ishchenko, G. V. Golubtsov, S. Dieckhöfer, W. Schuhmann and J. Masa, Catal. Today, , DOI:10.1016/j.cattod.2019.02.047.

R. Wang, Q. Dong, H. Wang, S. Ji, X. Wang and V. Linkov, Chem. - A Eur. J., 2020, chem.201905268.

Z. Yan, Q. Hu, G. Yan, H. Li, K. Shih, Z. Yang, X. Li, Z. Wang and J. Wang, Chem. Eng. J., 2017, 321, 495-501.

P. Lukanov, V. K. Anuganti, Y. Krupskaya, A.-M. Galibert, B. Soula, C. Tilmaciu, A. H. Velders, R. Klingeler, B. Büchner and E. Flahaut, Adv. Funct. Mater., 2011, 21, 3583-3588. Y. Xu, M. Mahmood, Z. Li, E. Dervishi, S. Trigwell, V. P. Zharov, N. Ali, V. Saini, A. R. Biris, D. Lupu, D. Boldor and A. S. Biris, Nanotechnology, 2008, 19, 435102.

O. N. Martyanov, V. F. Yudanov, R. N. Lee, S. A. Nepijko, H. J. Elmers, C. M. Schneider and G. Schönhense, Appl. Phys. A, 2005, 81, 679-683.

Y. V. Knyazev, A. I. Chumakov, A. A. Dubrovskiy, S. V. Semenov, I. Sergueev, S. S. Yakushkin, V. L. Kirillov, O. N. Martyanov and D. A. Balaev, Phys. Rev. B, 2020, 101, 094408.

A. A. Dubrovskiy, D. A. Balaev, K. A. Shaykhutdinov, O. A. Bayukov, O. N. Pletnev, S. S. Yakushkin, G. A. Bukhtiyarova and O. N. Martyanov, J. Appl. Phys., 2015, 118, 213901. S. N. Sidorov, L. M. Bronstein, V. A. Davankov, M. P. Tsyurupa, S. P. Solodovnikov, P. M. Valetsky, E. A. Wilder and R. J. Spontak, Chem. Mater., 1999, 11, 3210-3215. I. S. Edelman, E. A. Petrakovskaja, D. A. Petrov, S. M. Zharkov, R. I. Khaibullin, V. I. Nuzhdin and A. L. Stepanov, Appl. Magn. Reson., 2011, 40, 363-375.

A. C. Gossard, A. M. Portis, M. Rubinstein and R. H. Lindquist, Phys. Rev., 1965, 138, A1415-A1421.

V. V. Matveev, D. A. Baranov, G. Y. Yurkov, N. G. Akatiev, I. P. Dotsenko and S. P. Gubin, Chem. Phys. Lett., 2006, 422, 402-405.

A. S. Andreev, O. B. Lapina and S. V. Cherepanova, Appl. Magn. Reson., 2014, 45, 1009-1017.

A. S. Andreev, J.-B. d'Espinose de Lacaillerie, O. B. Lapina and A. Gerashenko, Phys. Chem. Chem. Phys., 2015, 17, 14598-14604.

Y. Liu, J. Luo, Y. Shin, S. Moldovan, O. Ersen, A. Hébraud, G.

Schlatter, C. Pham-Huu and C. Meny, Nat. Commun., 2016, 7, 11532.

M. A. Kazakova, A. S. Andreev, A. G. Selyutin, A. V. Ishchenko, A. V. Shuvaev, V. L. Kuznetsov, O. B. Lapina and J.-B. d'Espinose de Lacaillerie, Appl. Surf. Sci., 2018, 456, 657-665.

A. S. Andreev, M. A. Kazakova, A. V. Ishchenko, A. G. Selyutin, O. B. Lapina, V. L. Kuznetsov and J.-B. d'Espinose de Lacaillerie, Carbon N. Y., 2017, 114, 39-49.

M. A. Kazakova, V. L. Kuznetsov, S. N. Bokova-Sirosh, D. V. Krasnikov, G. V. Golubtsov, A. I. Romanenko, I. P. Prosvirin, A. V. Ishchenko, A. S. Orekhov, A. L. Chuvilin and E. D. Obraztsova, Phys. status solidi, 2018, 255, 1700260. H. P. Boehm, Carbon N. Y., 1994, 32, 759-769. A. Zhdanov and M. Kazakova (Shuvaeva), J. Anal. Chem., 2020, 75, 239-247.

Y. Liu, B. de Tymowski, F. Vigneron, I. Florea, O. Ersen, C. Meny, P. Nguyen, C. Pham, F. Luck and C. Pham-Huu, ACS Catal., 2013, 3, 393-404.

P. Panissod, J. P. Jay, C. Meny, M. Wojcik and E. Jedryka, Hyperfine Interact., 1996, 97-98, 75-98.

I. S. Oliveira and A. P. Guimarães, J. Magn. Magn. Mater., 1997, 170, 277-284.

M. B. Stearns, Phys. Rev., 1967, 162, 496-509.

D. L. Leslie-Pelecky and R. D. Rieke, Chem. Mater., 1996, 8, 1770-1783.

A. C. Gossard, A. M. Portis, M. Rubinstein and R. H. Lindquist, Phys. Rev., 1965, 138, A1415-A1421.

L. Neel, Ann. Geofis., 1949, 5, 99.

L. M. Liz-Marzán, M. Giersig and N. A. T. O. S. A. Division, Springer Netherlands, 2003.

R. M. Bozorth, Phys. Rev., 1954, 96, 311-316.

D. S. Rodbell, Phys. Phys. Fiz., 1965, 1, 279-305.

S. Ram, Mater. Sci. Eng. A, 2001, 304-306, 923-927. 